\title{
Apelin/APJ axis improves angiotensin II-induced endothelial cell senescence through AMPK/SIRT1 signaling pathway
}

Rongfeng Yang ${ }^{1,2}$, Wu Fang 3 , Jiawen Liang ${ }^{1}$, Chao Lin'1 ${ }^{1}$ Shaoyun $\mathrm{Wu}^{1}$, Shaodi Yan ${ }^{2}$, Chengheng $\mathrm{Hu}^{1}$, Xiao $\mathrm{Ke}^{2}$

${ }^{1}$ Department of Cardiology, First Affiliated Hospital of Sun Yat-sen University, Guangzhou, China

${ }^{2}$ Department of Cardiology, Shenzhen Sun Yat-sen Cardiovascular Hospital, Shenzhen, China

${ }^{3}$ Department of Geriatric, First Affiliated Hospital of Sun Yat-sen University, Guangzhou, China

Submitted: 15 July 2017

Accepted: 3 September 2017

Arch Med Sci 2018; 14, 4: 725-734

DOI: https://doi.org/10.5114/aoms.2017.70340

Copyright (c) 2017 Termedia \& Banach

\section{Abstract}

Introduction: Previous studies have shown that endothelial cell senescence is involved in cardiovascular diseases such as cardiac fibrosis, atherosclerosis and heart failure. Accumulating evidence indicates that apelin exerts protective effects on ageing-related endothelial dysfunction. In this study, we aim to investigate the role of the apelin/APJ axis in angiotensin II (AngII)-induced endothelium senescence and its associated mechanisms.

Material and methods: Senescence-related $\beta$-gal activity assay and western blot were used to evaluate human umbilical vein endothelial cell (HUVEC) senescence. In addition, DCFH-DA staining was carried out to detect the generation of reactive oxygen species (ROS). A validated, high-sensitivity real-time quantitative telomeric repeat amplification protocol (RQ-TRAP) was applied to determine telomerase activity in HUVECs, and a CCK-8 assay was employed to measure cellular viability.

Results: Angll induced an increase in SA- $\beta$-Gal-positive cells and upregulation on expression of P21 and PAI- 1 compared to the control group $(p<0.05)$, while apelin against this process $(p<0.05)$. The protective effects were attenuated when APJ, AMPK and SIRT1 expression was knocked down $(p<0.05)$. Furthermore, apelin reduced Angll-induced ROS generation and enhanced telomerase activity in HUVECs $(p<0.05)$, which contributed to increased HUVEC viability as assessed by the CCK- 8 assay $(p<0.05)$.

Conclusions: The apelin/APJ axis improved Angll-induced HUVEC senescence via the AMPK/SIRT1 signaling pathway, and the underlying mechanisms might be associated with reduced ROS production and enhanced telomerase activity.

Key words: apelin, endothelium, senescence, mechanisms.

\section{Introduction}

Ageing is an important and independent risk factor for cardiovascular diseases, which is considered at least partially due to ageing-related structural and functional alterations of the cardiovascular system [1]. Accumulating evidence has consistently demonstrated that ageing-related

\author{
Corresponding authors: \\ Xiao Ke \\ Department of Cardiology \\ Shenzhen Sun Yat-sen \\ Cardiovascular Hospital \\ 1021 Dongmen Bei Road \\ Shenzhen 518020 \\ Guangdong Province, China \\ Phone: +86 75525509566 \\ Fax: +8675525509566 \\ E-mail: \\ kexiao131131@163.com \\ Chengheng $\mathrm{Hu}$ \\ Department of Cardiology \\ The First Affiliated Hospital \\ of Sun Yat-sen University \\ 58 Zhongshan Road \\ Guangzhou 510080, China \\ Phone: +86 2087755766 , \\ ext. 8164 \\ Fax: +86 2087755766 , \\ ext. 8756 \\ E-mail: \\ huchengheng138@163.com
}


endothelial dysfunction plays a major role in cardiovascular disease development [2-4]. Apelin is a novel adipocyte-derived factor which has been identified as an endogenous ligand for the G protein-coupled receptor APJ [5]. In the cardiovascular system, apelin is predominantly expressed in endothelial cells, and the APJ receptor is expressed on endothelial cells, smooth muscle cells and cardiomyocytes [6]. Several active isoforms of apelin have been identified to date. Notably, a shorter peptide which consists of 13 amino acids exhibits the greatest activity [7]. Previous studies have revealed that apelin can promote angiogenesis and reduce ischemia-induced myocardial injury $[8,9]$. Recently, some studies have also shown that the apelin/APJ pathway is beneficial for improving cardiac fibrosis [10]. In addition, in patients with heart failure, plasma apelin level is decreased while an increased plasma apelin level appears to improve left ventricular remodeling [11, 12]. Taken together, previous findings indicate that the apelin/APJ pathway is involved in the alterations of cardiovascular structure.

Prior studies have revealed that endothelium ageing is associated with endothelial dysfunction and atherogenesis [13-15], and activation of the apelin/APJ pathway appears to ameliorate and improve these pathological processes $[9,10]$. However, the underlying mechanism is still elusive. There is a high homology between APJ receptor and angiotensin II receptor type I (AT1R), which shares identity of $54 \%$ in the transmembrane regions [16]. However, substantial evidence has shown that the apelin/APJ pathway has opposing actions to AngII/AT1R in regulation of the cardiovascular system. Meanwhile, a prior study indicated that Angll promoted the onset of senescence of endothelial cell [17]. In the present study, we therefore hypothesized that the apelin/APJ pathway might antagonize the Angll-induced endothelium senescence. Apelin is highly conserved among different species, and the C-terminal 13 amino acids (65-77) are completely conserved across all species studied [16], indicating that apelin-13 should be the best representative agent for experimental research. Therefore, in the current study, we used apelin-13 as a representative of apelin to investigate the above hypothesis.

\section{Material and methods}

\section{Cells culture and treatment}

Human umbilical vascular endothelial cells (HUVECS) were purchased from the Shanghai Institute for Biological Sciences, Chinese Academy of Sciences. HUVECS were cultured in endothelial cell medium (ECM) (Gibco, USA) supplemented with $10 \%$ fetal bovine serum (FBS, Gibco, USA),
$100 \mathrm{U} / \mathrm{ml}$ penicillin, $100 \mathrm{mg} / \mathrm{ml}$ streptomycin and $20 \mathrm{mg} / \mathrm{ml}$ vascular endothelial growth factor (VEGF). The cells were incubated at $37^{\circ} \mathrm{C}$ in a humidified environment with $5 \% \mathrm{CO}_{2}$. HUVECs with the concentration of $10^{5}$ per well were pre-treated with apelin-13 (10-8 M) (Sigma, USA) for $1 \mathrm{~h}$ and then treated with Angll $\left(10^{-6} \mathrm{M}\right)$ (Sigma, USA) for $24 \mathrm{~h}$. The medium was not changed during culture and treatment.

\section{RNA interference}

In the following experiment, the APJ, AMPK and SIRT1 genes of HUVECs were knocked out using RNA interference. In brief, the HUVECs (with 70\% confluence) were transfected with a plasmid containing siRNAs specific against the APJ, AMPK and SIRT1 genes and the scramble control for $48 \mathrm{~h}$ in accordance with the manufacturer's instructions. The siRNAs were synthesized and purchased from RiboBio Company (China). The short hairpin RNA sequences were as follows: SiAMPK-1: 5'-GAGGAGAGCTATTTGATTA-3'; siAMPK-2: 5'-GCAGAAGTATGTAGAGCAA-3'; siAMPK-3: 5'-ACACATGAATGCAAAGATA-3'. Meanwhile, three short hairpin RNA vectors were constructed to encode sequences targeting human SIRT1 mRNA (siSIRT1-001: 5'-GCTAAGAATTTCAGGATTA-3' and siSIRT1-002: 5'-GGAAATATATCCTGGACAA-3' and siSIRT1-003: 5'-CCTTAAAACTAGAGATCAA-3'). Thereafter, the HUVECs were harvested and used for the following experiments.

\section{Senescence-associated $\beta$-gal activity assay}

Senescence was detected using a senescenceassociated $\beta$-galactosidase (SA- $\beta$-gal)-positive approach. The HUVECs were stained with 4',6-diamino-phenylindole (DAPI) $(0.2 \mu \mathrm{g} / \mathrm{ml}$ in $10 \mathrm{mmol} / \mathrm{l}$ Tris- $\mathrm{HCl}, \mathrm{pH} 7.0,10 \mathrm{mmol} / \mathrm{l}$ EDTA, $100 \mathrm{mmol} / \mathrm{l}$ $\mathrm{NaCl}$ ) for $10 \mathrm{~min}$ and then the total number of HUVECs was counted. The cells were imaged under a bright-field microscope at a magnification of 200x. The senescent cells were observed in an optical microscope and counted from 5 random fields of vision.

\section{Western blot}

After treatments, the HUVECs were harvested and lysed with cell lysis solution at $4^{\circ} \mathrm{C}$ for $30 \mathrm{~min}$. Loading buffer was added to cytosolic extracts, and after boiling for about $5 \mathrm{~min}, 30 \mu \mathrm{g}$ of protein of each sample were resolved by $10 \%$ sodium dodecyl sulfate-polyacrylamide gel electrophoresis (SDS-PAGE), and then the total gel was transferred into polyvinylidene fluoride (PVDF) membranes. The membranes were probed with anti-P21 (CST 2947), anti-PAI-1 (CST 11907), anti-APJ Receptor (AB 214369), anti-t-AMPK (CST 5832), antip-AMPK (CST 2535), anti-SIRT1 (CST 2493) and 
anti-GAPDH (GXP 199629) primary antibodies overnight at $4^{\circ} \mathrm{C}$ with $1: 1000$ dilution followed by horseradish peroxidase-conjugated secondary antibodies (goat anti-rabbit IgG). The immunoreactive signals were visualized by enhanced chemiluminescence $(E C L)$ detection. In order to quantify the protein expression, the X-ray films were scanned and analyzed with Image J $1.47 \mathrm{i}$ software.

\section{Measurement of intracellular reactive oxygen species generation}

Intracellular reactive oxygen species (ROS) generation was tested by the oxidative conversion of cell-permeable oxidation of $2^{\prime}, 7^{\prime}$-dichlorodihydrofluorescein diacetate (DCFH-DA) to fluorescent DCF. The HUVECs were cultured on 6-well plates with ECM. With different treatments, each well was washed 3 times with PBS, and $10 \mu \mathrm{M} \mathrm{DCFH-}$ DA solution in serum-free medium was added to the wells, and then the HUVECs were incubated at $37^{\circ} \mathrm{C}$ for a further $30 \mathrm{~min}$ in an incubator. After that, the cells were washed 5 times with PBS, and DCF fluorescence was measured over the entire field of vision using a fluorescence microscope connected to an imaging system (BX50-FLA, Olympus, Tokyo, Japan). The mean fluorescence intensity (MFI) of ROS from 5 random fields was measured using Image J $1.47 i$ software and the MFI was used as an index of the amount of ROS. $\mathrm{MFI}$ in the Angll group was considered as $100 \%$ for comparison with other groups.

\section{Real-time quantitative telomeric repeat amplification protocol (RQ-TRAP) assay}

Telomerase activity in 1,000 HUVECs was assessed using a SYBR Green (Applied Biosystems, Foster City, CA) RQ-TRAP assay as described by Henning Wege [14]. The threshold cycle value (Ct) of each sample was compared with standard curves generated from serial dilutions of telomerase-positive 293T cell extracts (1000, 100, 10 and 1 cells). Standards and negative controls with heat-inactivated samples and lysis buffer were assayed on each plate. Each sample was analyzed and the telomerase activity was calculated relative to 293T cells. Telomerase activity in the control group was considered as $100 \%$ for comparison with other groups.

\section{Evaluation of HUVEC viability}

The HUVECs were seeded on 96-well plates at a density of $1 \times 10^{4}$ cells/well, and the CCK- 8 assay was employed to measure cellular viability. After the indicated treatments, $10 \mu \mathrm{l}$ of CCK-8 solution at a $1: 10$ dilution was added to every well and then the plate was incubated for $3 \mathrm{~h}$ in the incubator. Absorbance at $450 \mathrm{~nm}$ was measured with a microplate reader (Molecular Devices, Sunny- vale, CA, USA), and the means of optical density (OD) of 4 wells in the indicated groups were used to calculate the percentage of cell viability in relation to the control group, which was based on the following formula: cell viability $(\%)=(O D$ treatment group/OD control group) $\times 100 \%$.

\section{Statistical analysis}

All data were presented as the mean \pm SD. Differences between groups were analyzed by oneway analysis of variance (ANOVA) using the SPSS 19.0 software (SPSS, Chicago, IL, USA), followed by the LSD post hoc between-group comparison test. $P<0.05$ was considered to be statistically significant.

\section{Results}

\section{Apelin inhibited Angll-induced HUVEC senescence and intracellular ROS production}

To assess the onset of senescence, senescence-associated $\beta$-galactosidase was detected. As shown in Figure $1 \mathrm{~A}$, Angll induced an increase in SA- $\beta$-Gal-positive cells compared to the control group, demonstrating that HUVEC senescence was successfully induced by Angll. Of note, SA- $\beta$ Gal-positive cells were significantly reduced by apelin. We further tested the effects of apelin on the expression of p21 proteins in Angll-treated cells since $\mathrm{P} 21$ protein has been shown to increase during cell senescence. As shown in Figure $1 \mathrm{~B}$, p21 expression in the apelin + Angll group was significantly lower compared to the Angll group.

It has been well documented that cell senescence is closely associated with intracellular ROS production [18]. Therefore, we also tested the changes of ROS with and without apelin treatment using the H2DCF-DA labeling assay. As shown in Figure $1 \mathrm{C}$, Angll remarkably increased the H2DCF-DA oxidation level in HUVECs, while treatment with apelin significantly inhibited ROS production.

\section{Apelin/APJ inhibited Angll-induced cell senescence via AMPK/SIRT1 pathway}

To determine whether the APJ/AMPK/SIRT1 pathway was involved in apelin-mediated HUVEC senescence improvement, a non-silencing scrambled control (SCR), siAPJ, SiAMPK and siSIRT1 were used. After transfection of HUVECs with APJ siRNA, expression of APJ was determined using western blot and the results showed satisfactory transfection efficiency. As shown in Figure 2 A, siAMPK-2 significantly reduced t-AMPK expression. In contrast, siAMPK-1 and siAMPK-3 did not alter t-AMPK expression. In addition, we found that SIRT1 protein was also significantly decreased by SiSIRT1-2. 

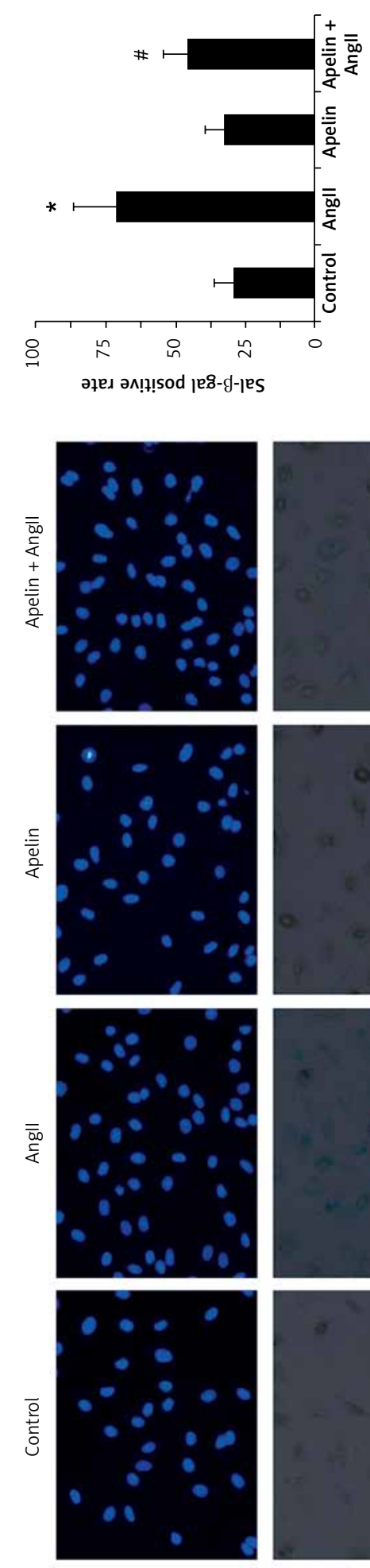

$\varangle$

$I d \forall O$
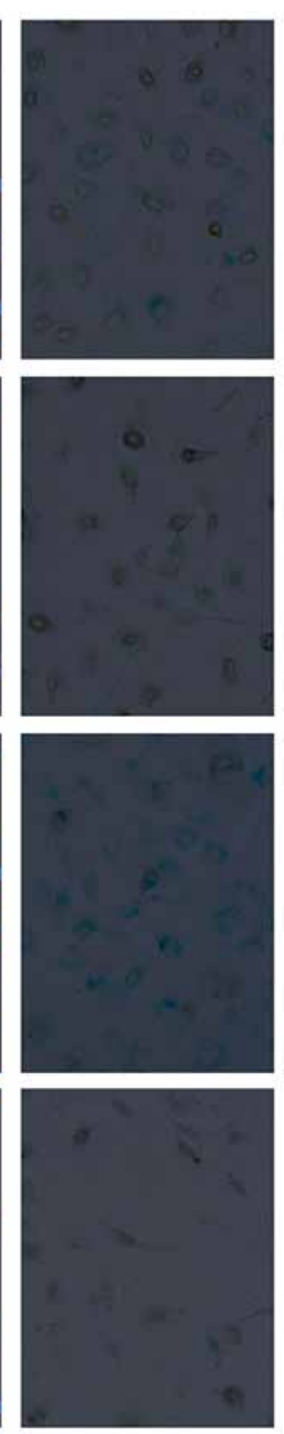

ןe8-g-jes
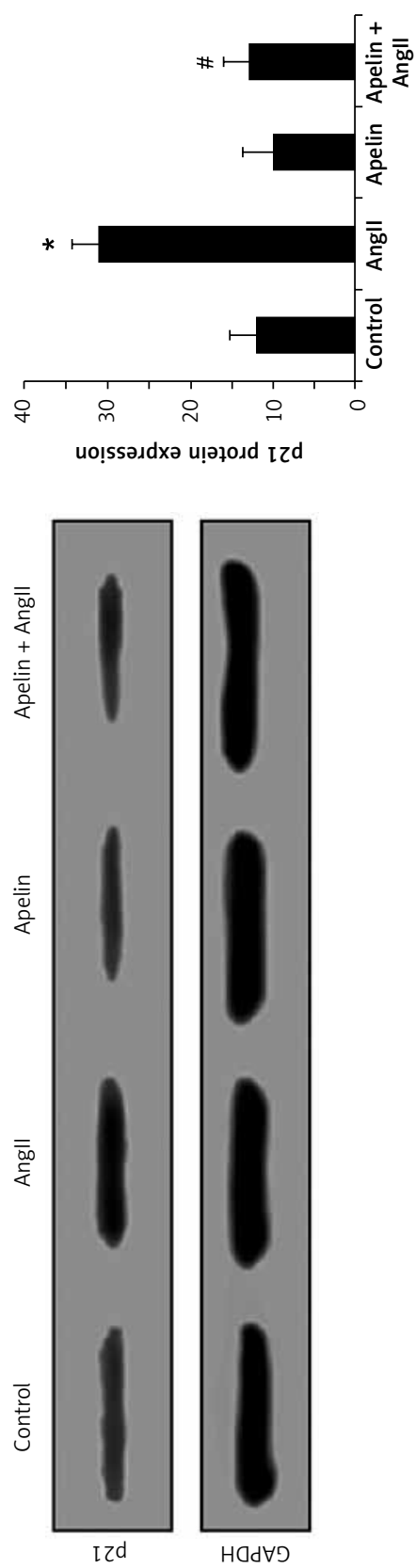

a

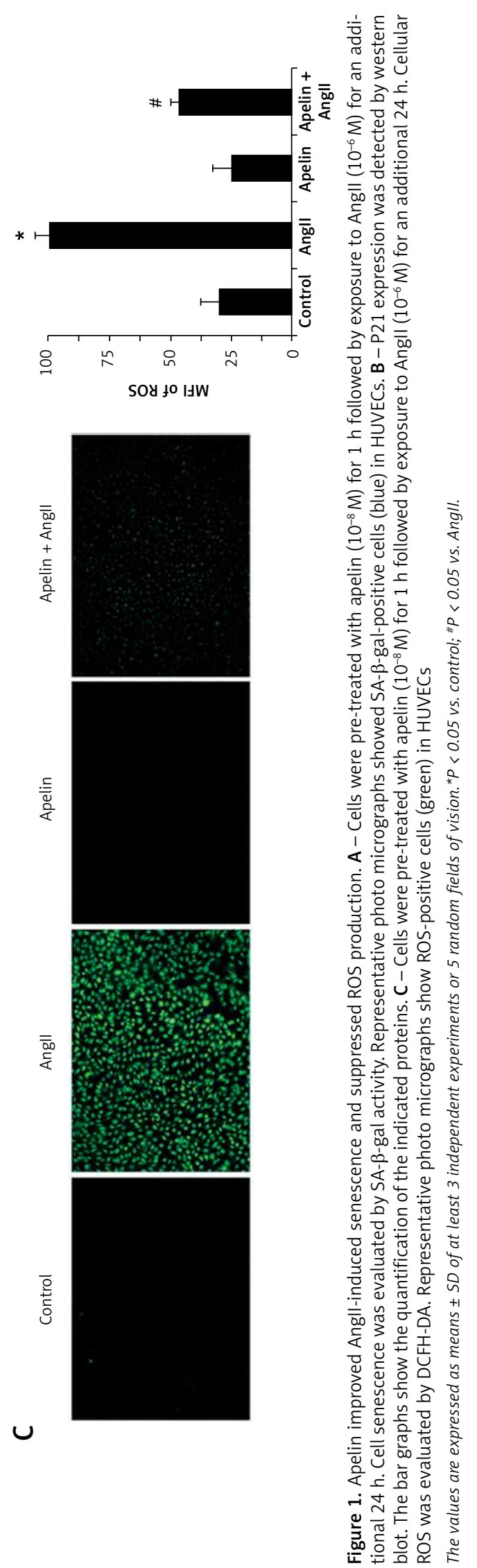


We further investigated whether APJ, AMPK and SIRT1 were associated with improvement of HUVEC senescence by apelin treatment. As shown in Figure $2 \mathrm{~B}$, the expression levels of APJ, p-AMPK and SIRT1 were significantly increased by apelin treatment while PAI-1 protein was reduced. Furthermore, APJ knockdown led to an increase in PAI-1 expression and a decline in AMPK and SIRT1
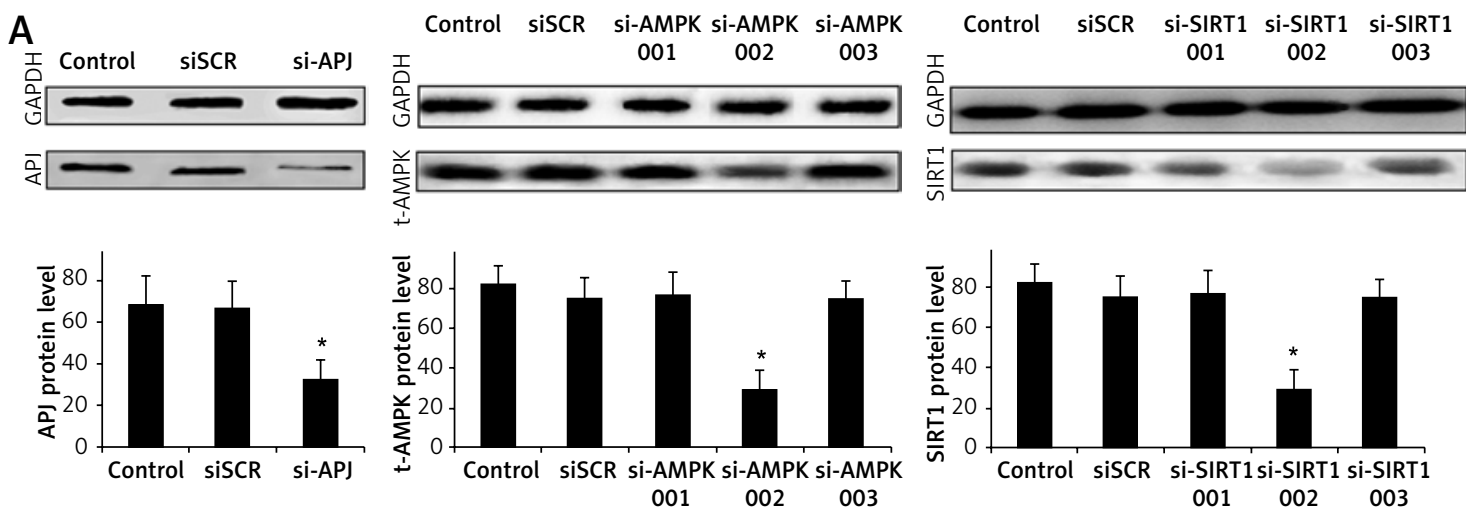

B
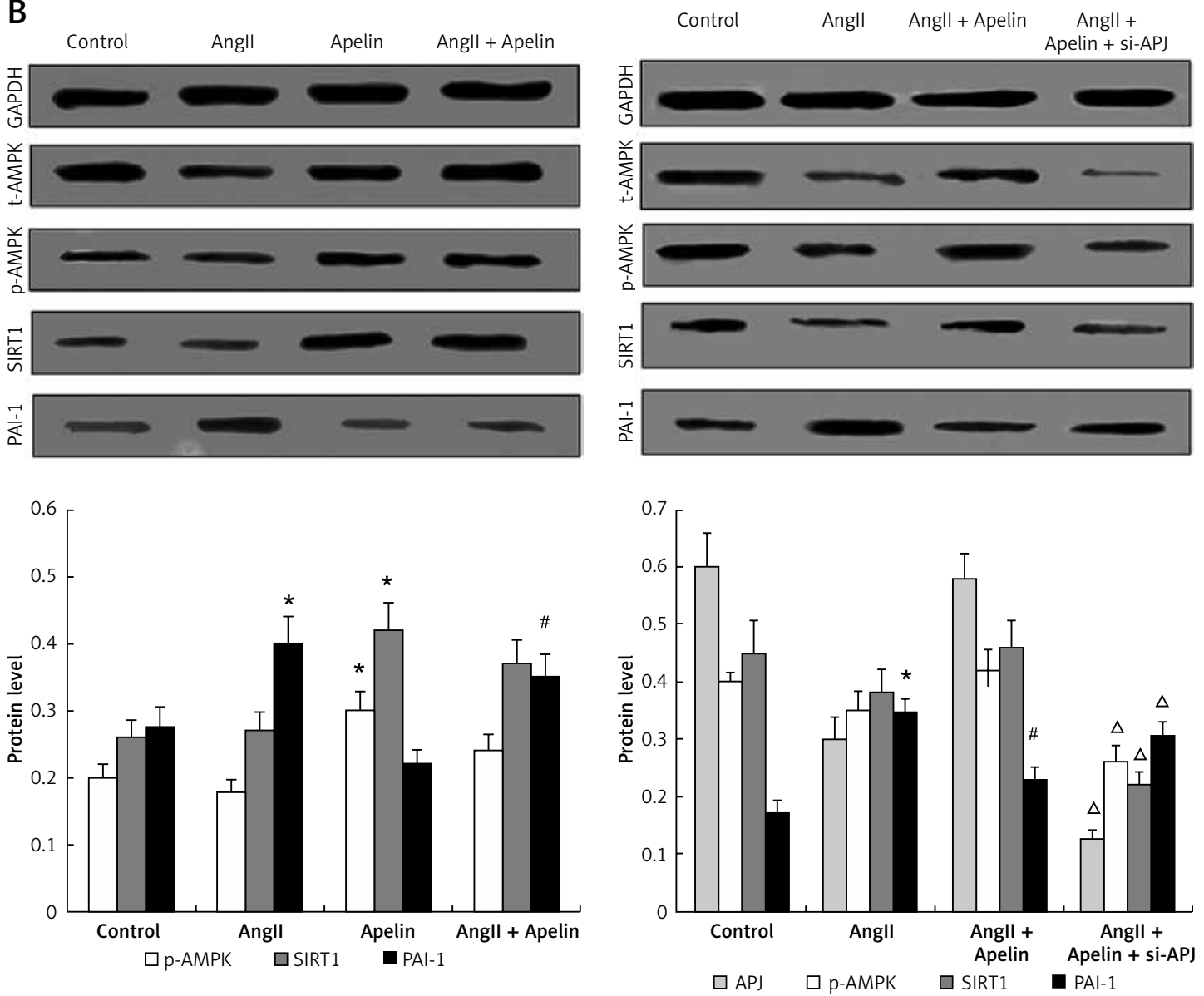

Figure 2. Apelin/APJ inhibited Angll-induced senescence through AMPK/SIRT1 signal pathway. A - SIRT1, AMPK, APJ were respectively knocked down by siRNA transfection as described in the Material and methods section. APJ, AMPK and SIRT1 expression was detected by western blot. B - As shown in the left panel, cells were pre-treated with apelin $\left(10^{-8} \mathrm{M}\right)$ for $1 \mathrm{~h}$ followed by exposure to Angll $\left(10^{-6} \mathrm{M}\right)$ for an additional $24 \mathrm{~h}$. Western blot analysis was performed. As shown in the right panel, APJ was knocked down. At $48 \mathrm{~h}$ after transfection, the cells were pre-treated with apelin $\left(10^{-8} \mathrm{M}\right)$ for $1 \mathrm{~h}$ followed by exposure to Angll $\left(10^{-6} \mathrm{M}\right)$ for an additional $24 \mathrm{~h}$. Western blot analysis was performed

The values are expressed as means $\pm S D$ of at least 3 independent experiments. ${ }^{*} P<0.05$ vs. control; ${ }^{*} p<0.05$, vs. Angll; ${ }^{\Delta} p<0.05$, vs. apelin + Angll. 

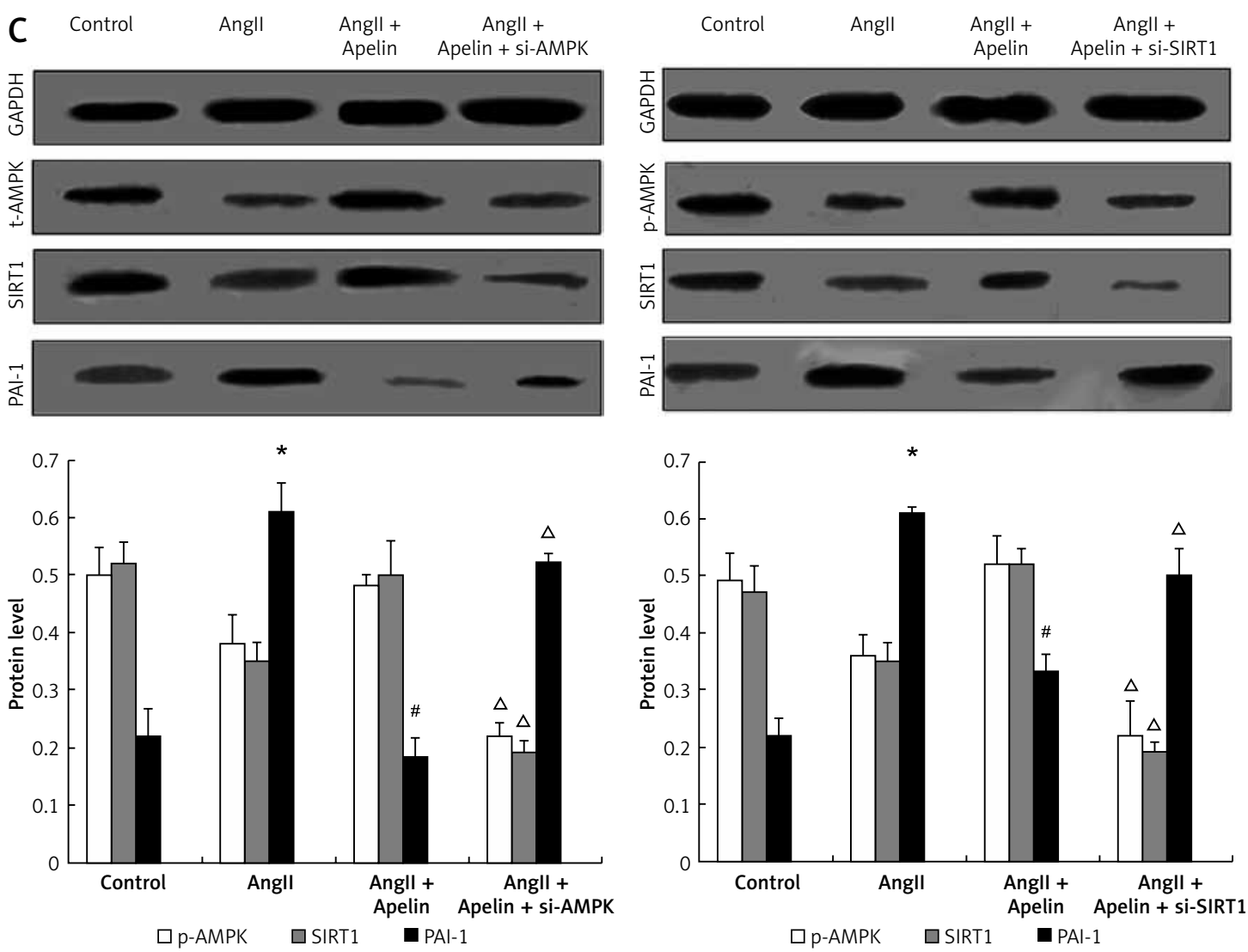

Figure 2. Cont. C - As shown in the left panel, AMPK was knocked down. At $48 \mathrm{~h}$ after transfection, the cells were pre-treated with apelin $\left(10^{-8} \mathrm{M}\right)$ for $1 \mathrm{~h}$ followed by exposure to Angll $\left(10^{-6} \mathrm{M}\right)$ for an additional $24 \mathrm{~h}$. Western blot analysis was performed. As shown in the right panel, SIRT1 was knocked down. At $48 \mathrm{~h}$ after transfection, the cells were pre-treated with apelin $\left(10^{-8} \mathrm{M}\right)$ for $1 \mathrm{~h}$ followed by exposure to Angll $\left(10^{-6} \mathrm{M}\right)$ for an additional $24 \mathrm{~h}$. Western blot analysis was performed. The bar graphs show the quantification of the indicated protein

The values are expressed as means $\pm S D$ of at least 3 independent experiments. ${ }^{*} P<0.05$ vs. control; ${ }^{*} p<0.05, v s$. Angll; ${ }^{\Delta} p<0.05$, vs. apelin + Angll.

expression, indicating that APJ might be an upstream regulator of the AMPK/SIRT1 pathway. As shown in Figure 2 C, PAI-1 reduction by apelin was also abrogated by SIAMPK-2 and siSIRT1-2, indicating that the apelin/APJ axis improved Angll-induced cell senescence via the AMPK/ SIRT1 pathway.

\section{Apelin/APJ inhibited Angll-induced HUVEC senescence and intracellular ROS production via the AMPK/SIRT1 pathway}

As shown in Figure $3 \mathrm{~A}$, compared to the Angll group, addition of apelin reduced SA- $\beta$-Gal-positive cells. Of note, as shown in Figure 3 B APJ, AMPK and SIRT1 knockdown resulted in a significant increase in H2DCF-DA labeled cells, indicating that apelin's reduction of Angll-induced ROS production was associated with the AMPK/SIRT1 pathway.

\section{Effects of apelin on telomerase activity in Angll-treated HUVEC}

Initiation and development of endothelium senescence is known to be influenced by telomerase activity [19]. Therefore, we further tested whether treatment with apelin was capable of regulating telomerase activity in Angll-treated HUVECs. As presented in Figure $4 \mathrm{~A}$, Angll significantly diminished telomerase activity. However, this effect was significantly reduced by treatment with apelin. Of note, treatment with siAPJ, siSIRT1 and siAMPK decreased telomerase activity. In addition, we also examined whether increased telomerase activity with apelin treatment was associated with improvement of cellular viability. As presented in Figure $4 \mathrm{~B}$, apelin improved cell viability as indexed by CCK-8, and this protective effect was abolished by APJ, AMPK and SIRT1 knockdown.

\section{Discussion}

In the present study, we provide new evidence that apelin reduced Angll-induced HUVEC senescence, which in turn improved cellular viability. Importantly, we also demonstrated for the first time that these cellular protections were associated with the AMPK/SIRT1 signaling pathway (as presented in Figure 5). Moreover, our data sug- 


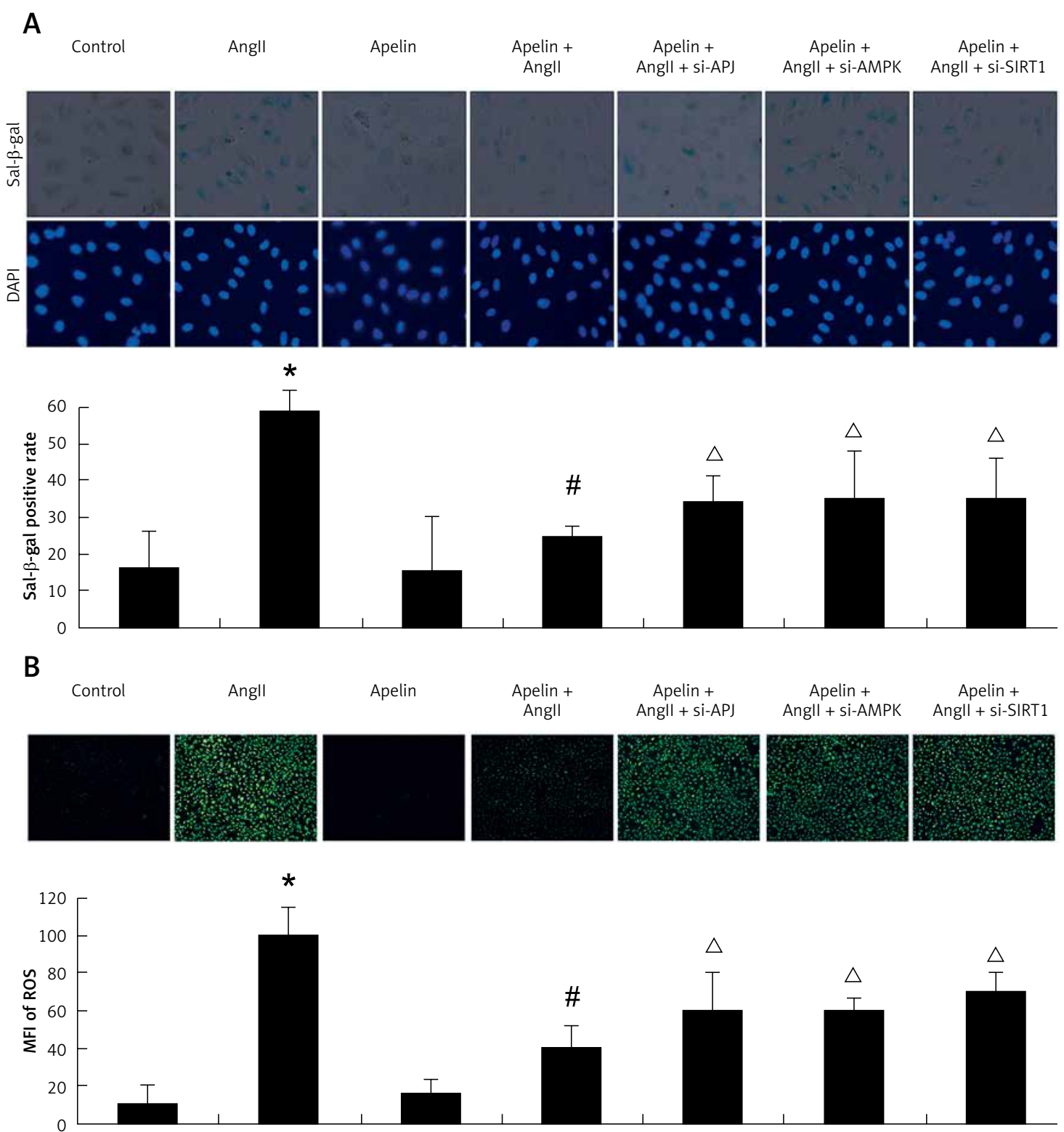

Figure 3. Apelin improved Angll-induced ageing and reduced ROS production via AMPK/SIRT1 signaling pathway. A - SIRT1, AMPK, APJ were knocked down by siRNA transfection. At $24 \mathrm{~h}$ after transfection, the cells were treated with apelin $\left(10^{-8} \mathrm{M}\right)$ for $1 \mathrm{~h}$ followed by exposure to Angll $\left(10^{-6} \mathrm{M}\right)$ for an additional $24 \mathrm{~h}$. Cell senescence was evaluated by SA- $\beta$-gal activity. Representative photomicrographs show SA- $\beta$-gal-positive cells (blue) in HUVECs. B - The level of intracellular ROS was assessed. Representative photomicrographs show ROS-positive cells (green) in HUVECS

The values are expressed as means $\pm S D$ of at least 3 independent experiments or 5 random fields of vision. ${ }^{*}<0.05$ vs. control; ${ }^{\#} p<0.05$ vs. Angll; ${ }^{\Delta} p<0.05$ vs. apelin + Angll.

gested that ROS suppression and telomerase activation were also involved in these pathological alterations.

It is well known that endothelium senescence causes endothelial dysfunctional and ageing-related vascular disorders such as atherosclerosis [13]. A prior study revealed that Angll induced atherosclerosis by promoting vascular ageing [20]. It is also reported that Angll plays an important role in endothelium senescence [17]. It would be clinically relevant to improve Angll-induced endothelium senescence. It has been found that the
apelin/APJ axis exerts the opposite physiological effects of AngII/AT1R. Lee et al. reported that apelin had hypotensive properties, which was in contrast to the vasopressor effects of angiotensin II [16]. Moreover, apelin reduced Angll-induced cardiovascular fibrosis and atherosclerosis [21, 22]. Interestingly and importantly, our current study showed that the apelin/APJ axis was involved in the ageing-related pathological alterations of HUVECs. This finding indicates that the apelin/APJ axis may play an important role in the regulation of endothelial dysfunction. 
A

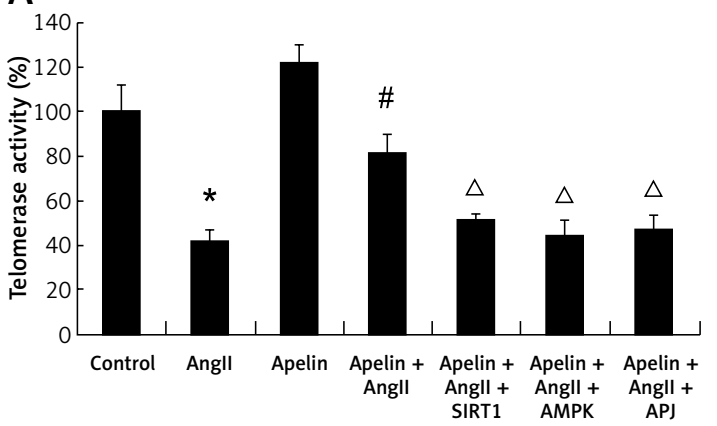

B

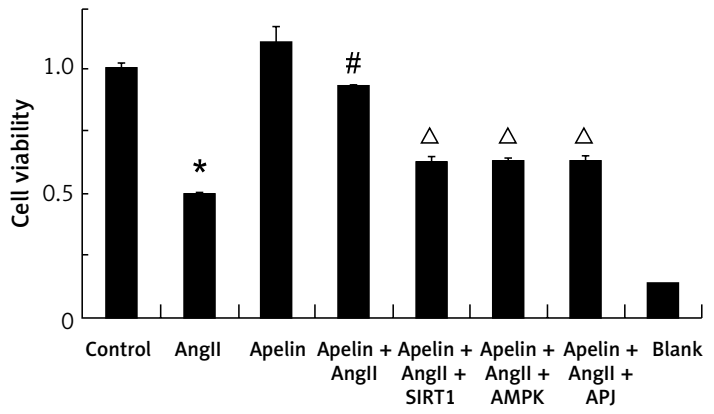

Figure 4. Apelin promoted telomerase activity in Angll-treated HUVEC. A - SIRT1, AMPK, APJ were knocked down by siRNA transfection. At $24 \mathrm{~h}$ after transfection, the cells were treated with apelin $\left(10^{-8} \mathrm{M}\right)$ for $1 \mathrm{~h}$ followed by exposure to Angll $\left(10^{-6} \mathrm{M}\right)$ for an additional $24 \mathrm{~h}$. Telomerase activity was investigated by RQ-TRAP. Telomerase activity in the control group was considered as $100 \%$ compared to the other groups. B - Cell viability was detected by CCK-8 assay. The bar charts show the cell viability of the indicated group

The values are expressed as means $\pm S D$ of at least 3 independent experiments. ${ }^{*} P<0.05$ vs. control; ${ }^{*} p<0.05$, vs. Angll; ${ }^{\Delta} p<0.05$, vs. apelin + Angll.

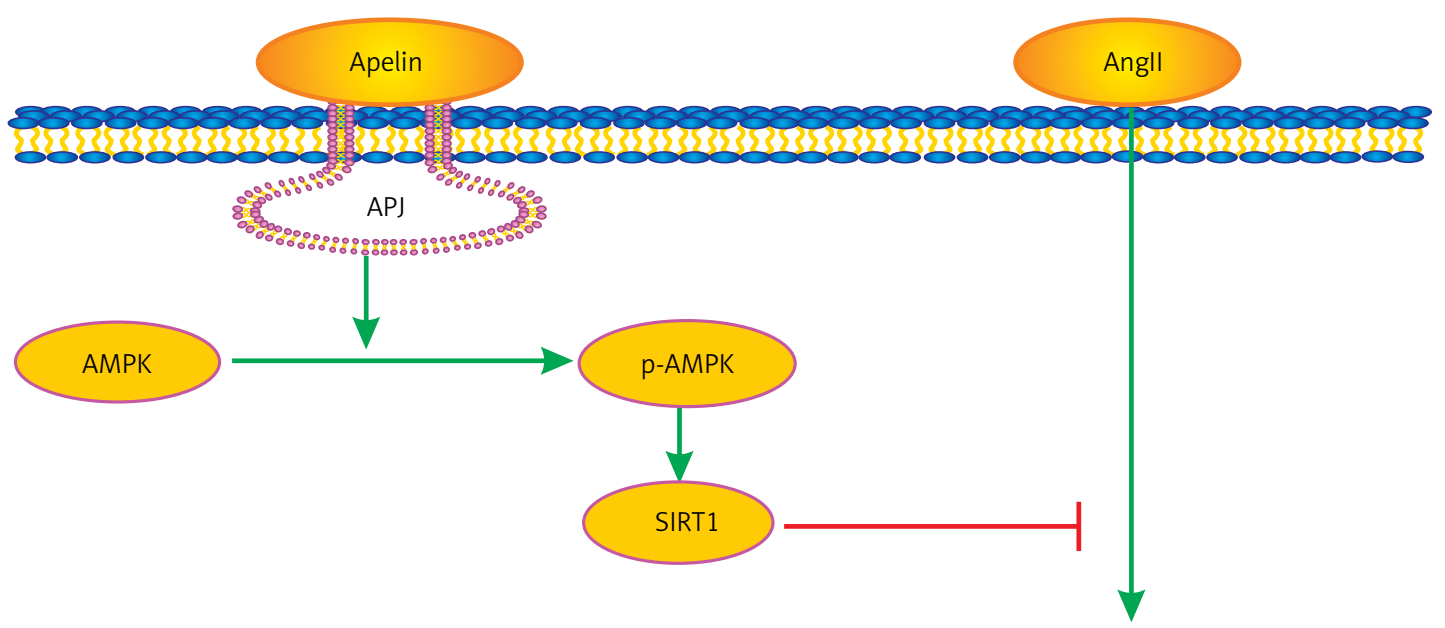

ROS activation

Telomerase suppression

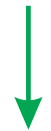

HUVESS (P21 PAI-1

Ageing sal- $\beta$-gal)

Figure 5. Pathway involved in the protective effect of apelin/APJ axis in Angll induced HUVECs ageing. Apelin/ APJ axis induces the phosphorylation of AMPK, causing SIRT1 activation. This process inhibits Angll-induced ROS generation and increases telomerase and finally improves Angll-induced HUVECs ageing

We have additionally investigated the underlying mechanisms of the endothelium-protective effects of the apelin/APJ axis. AMP-activated protein kinase (AMPK), a key regulator of metabolism, has been reported to be associated with ageing-related pathological changes in lower organisms such as yeast and worms [23, 24]. However, in higher organisms, the role of AMPK in the regulation of ageing-related changes is not clear yet. Some studies have reported that ageing increased AMPK activation [25], while others have reported decreased AMPK activity [26]. Previous studies have demonstrated that apelin can activate AMPK phosphorylation, which in turn inhibits the ageing-related cardiac fibrosis in animal models [10, 27], indicating a close connection between apelin and AMPK in ageing-related cardiovascular system changes. We observed that apelin-13 improved AMPK phosphorylation which in turn resulted in a reduction of Angll-induced HUVEC senescence. Notably, sirtuin 1 (SIRT1), an $\mathrm{NAD}^{+}$-dependent protein deacetylase regulated by AMPK, also has anti-ageing properties. Prior studies showed that SIRT1 improved ageing-related cardiovascular diseases by regulating a variety of cellular processes [28], and the AMPK/SIRT1 sig- 
naling pathway might play an important role in regulation of ageing. As expected, findings from our current study supported the notion that apelin attenuated Angll-induced HUVEC senescence through the AMPK/SIRT1 signaling pathway.

Improvement of ROS might be another mechanism. It has been reported that Angll-induced ROS generation in several pathophysiological processes such as vascular smooth muscle cell hypertrophy [29, 30]. Recent studies suggested that ROS generation accelerated the onset of cellular senescence [18, 31]. Moreover, Li et al. reported that Angll promoted cellular senescence and apoptosis via ROS [17]. We also found that apelin reduced ROS generation in Angll-treated HUVECS, which was abrogated by APJ knockout. In addition, AMPK and SIRT1 knockout also led to increased ROS generation, indicating that suppressing ROS generation might be associated with the endothelium-protective effects of the apelin/APJ axis.

It is generally believed that telomere length is a key factor in regulating cellular life span. Telomere shortening is like a molecular clock that triggers senescence. Telomerase, an RNA-directed DNA polymerase, is capable of maintaining the length of telomeres in endothelial cells and therefore regulates senescence [32]. A prior study showed that telomerase activation delayed endothelium senescence [33]. Therefore, it may be a new strategy for anti-ageing by regulating the telomerase activity. In this study, we investigated the role of telomerase activity in Angll-induced HUVEC senescence. Our data revealed that Angll diminished telomerase activity. However, apelin treatment enhanced telomerase activation and decreased HUVEC senescence. Telomerase activation induced by apelin was attenuated with APJ, AMPK and SIRT1 knockdown, indicating that apelin/APJ improved endothelium senescence through telomerase inactivation dependent on the AMPK/SIRT1.

In conclusion, our current study reveals that the apelin/APJ axis improved Angll-induced HUVEC senescence through the AMPK/SIRT1 signaling pathway, and the associated mechanisms might be related to decreased ROS production and increased telomerase activity. Our findings provide novel insights into the beneficial effects of apelin on ageing-related vascular diseases.

\section{Acknowledgments} thors.

Rongfeng Yang and Wu Fang are co-first au-

The study was supported by grants from the National Natural Science Foundation of China (NO. 81700263 and 81700323), the Natural Science Foundation of Guangdong Province of China (2016A030313248), the Science and Technology project of Guangzhou city of China (201604020129), and the Science and Technology project of Shenzhen city of China (JCYJ20170307161535847), and the study was supported by grants from the Research Project (Doctoral Innovation Program) of Health Planning system of Shenzhen city (SZBC2017007).

\section{Conflict of interest}

The authors declare no conflict of interest.

\section{References}

1. Barodka VM, Joshi BL, Berkowitz DE, Hogue CW Jr, Nyhan D. Review article: implications of vascular aging. Anesthesia Analgesia 2011; 112: 1048-60.

2. Tao J, Jin YF, Yang Z, et al. Reduced arterial elasticity is associated with endothelial dysfunction in persons of advancing age: comparative study of noninvasive pulse wave analysis and laser Doppler blood flow measurement. Am J Hypertens 2004; 17: 654-9.

3. Taddei S, Virdis A, Mattei P, et al. Aging and endothelial function in normotensive subjects and patients with essential hypertension. Circulation 1995; 91: 1981-7.

4. Virdis A, Ghiadoni L, Giannarelli C, Taddei S. Endothelial dysfunction and vascular disease in later life. Maturitas 2010; 67: 20-4.

5. Tatemoto K, Hosoya M, Habata Y, et al. Isolation and characterization of a novel endogenous peptide ligand for the human APJ receptor. Biochem Biophys Res Commun 1998; 251: 471-6.

6. Yang S, Li H, Tang L, et al. Apelin-13 protects the heart against ischemia-reperfusion injury through the RISK-GSK-3beta-mPTP pathway. Arch Med Sci 2015; 11 : 1065-73.

7. Lee DK, Ferguson SS, George SR, O'Dowd BF. The fate of the internalized apelin receptor is determined by different isoforms of apelin mediating differential interaction with beta-arrestin. Biochem Biophys Res Commun 2010; 395: 185-9.

8. Momiyama Y. Association between plasma apelin levels and coronary collateral development in patients with stable angina pectoris. Atherosclerosis 2014; 235: 349-50.

9. Novakova V, Sandhu GS, Dragomir-Daescu D, Klabusay M. Apelinergic system in endothelial cells and its role in angiogenesis in myocardial ischemia. Vascul Pharmacol 2016; 76: 1-10.

10. Huang S, Chen L, Lu L, Li L. The apelin-APJ axis: a novel potential therapeutic target for organ fibrosis. Clin Chim Acta 2016; 456: 81-8.

11. Chong KS, Gardner RS, Morton JJ, Ashley EA, McDonagh TA. Plasma concentrations of the novel peptide apelin are decreased in patients with chronic heart failure. Eur J Heart Fail 2006; 8: 355-60.

12. Francia P, Salvati A, Balla C, et al. Cardiac resynchronization therapy increases plasma levels of the endogenous inotrope apelin. Eur J Heart Fail 2007; 9: 306-9.

13. Minamino T, Miyauchi H, Yoshida T, Tateno K, Kunieda T, Komuro I. Vascular cell senescence and vascular aging. J Mol Cell Cardiol 2004; 36: 175-83.

14. Maizel J, Vasko R, Xavier S, Chen J, Cao J, Goligorsky MS. Vascular senescence and organ fibrosis. Proceedings of the Physiological Society 2013; Proc 37th IUPS.

15. Minamino T, Miyauchi H, Yoshida T, Ishida Y, Yoshida H, Komuro I. Endothelial cell senescence in human athero- 
sclerosis: role of telomere in endothelial dysfunction. Circulation 2002; 105: 1541-4.

16. Lee D, Cheng R, T, Fan T, et al. Characterization of apelin, the ligand for the APJ receptor. J Neurochem 2000; 74: 34-41.

17. Li P, Guo X, Lei P, Shi S, Luo S, Cheng X. PI3K/Akt/uncoupling protein 2 signaling pathway may be involved in cell senescence and apoptosis induced by angiotensin II in human vascular endothelial cells. Mol Biol Rep 2014; 41: 6931-7.

18. Mikhed Y, Daiber A, Steven S. Mitochondrial oxidative stress, mitochondrial DNA damage and their role in agerelated vascular dysfunction. Int J Mol Sci 2015; 16: 15918-53.

19. Minamino T, Komuro I. Role of telomere in endothelial dysfunction in atherosclerosis. Curr Opin Lipidol 2002; 13: 537-43.

20. Huang F, Thompson JC, Wilson PG, Aung HH, Rutledge JC, Tannock LR. Angiotensin II increases vascular proteoglycan content preceding and contributing to atherosclerosis development. J Lipid Res 2008; 49: 521-30.

21. Siddiquee K, Hampton J, Khan S, et al. Apelin protects against angiotensin II-induced cardiovascular fibrosis and decreases PAI-1 production. J Hypertens 2011; 29: 724-31.

22. Chun HJ, Ali ZA, Kojima Y, et al. Apelin signaling antagonizes Ang II effects in mouse models of atherosclerosis. J Clin Investig 2008; 118: 3343-54.

23. Pallottini V, Montanari L, Cavallini G, Bergamini E, Gori Z, Trentalance A. Mechanisms underlying the impaired regulation of 3-hydroxy-3-methylglutaryl coenzyme A reductase in aged rat liver. Mech Ageing Dev 2004; 125: 633-9.

24. Gonzalez AA, Kumar R, Mulligan JD, Davis AJ, Saupe KW. Effects of aging on cardiac and skeletal muscle AMPK activity: basal activity, allosteric activation, and response to in vivo hypoxemia in mice. Am J Physiol Regul Integr Comp Physiol 2004; 287: R1270.

25. Mulligan JD, Gonzalez AA, Kumar R, Davis AJ, Saupe KW. Aging elevates basal adenosine monophosphate-activated protein kinase (AMPK) activity and eliminates hypoxic activation of AMPK in mouse liver. J Gerontol 2005; 60: 21-7.

26. Reznick RM, Zong H, Li J, et al. Aging-associated reductions in AMP-activated protein kinase activity and mitochondrial biogenesis. Cell Metabol 2007; 5: 151-6.

27. Pchejetski D, Foussal C, Alfarano C, et al. Apelin prevents cardiac fibroblast activation and collagen production through inhibition of sphingosine kinase 1. Eur Heart 2012; 33: 2360-9.

28. Guarente L. Sirtuins, aging, and medicine. N Engl J Med 2011; 364: 2235-44

29. Wilson SK. Role of oxygen-derived free radicals in acute angiotensin II: induced hypertensive vascular disease in the rat. Circ Res 1990; 66: 722-34.

30. Ushiofukai M, Zafari AM, Fukui T, Ishizaka N, Griendling KK. p22phox Is a critical component of the superoxide-generating NADH/NADPH oxidase system and regulates angiotensin II-induced hypertrophy in vascular smooth muscle cells. J Biol Chem 1996; 271: 23317-21.

31. Adelibieke Y, Shimizu H, Muteliefu G, Bolati D, Niwa T. Indoxyl sulfate induces endothelial cell senescence by increasing reactive oxygen species production and p53 activity. J Renal Nutrition 2012; 22: 86-9.

32. Yang J, Chang E, Cherry AM, et al. Human endothelial cell life extension by telomerase expression. J Biol Chem 1999; 274: 26141-8.
33. Vasa M, Breitschopf K, Zeiher AM, Dimmeler S. Nitric oxide activates telomerase and delays endothelial cell senescence. Circ Res 2000; 87: 540-2. 